\title{
REMOVAL OF HEAVY METALS FROM REFINERIES WASTEWATER
}

\author{
"Zainab J. Shadhan'
}

Mohammed N. Abbas ${ }^{1}$

1) Environmental Engineering Department, College of Engineering, Mustansiriyah University, Baghdad, Iraq

\begin{abstract}
In this study, the adsorption method was investigated for removing vanadium $(\mathrm{V}+5)$, nickel $(\mathrm{Ni}+2)$ and cadmium $(\mathrm{Cd}+2)$ ions from aqueous solutions contaminated with these metals, which simulate the polluting metals of the liquid wastewater of oil refineries in three Iraqi refineries, namely the Kirkuk refinery Kirkuk governorate in northern Iraq, and the Doura refinery - Baghdad in central Iraq and Al-Shuaiba refinery in Basra Governorate, southern Iraq. Three types of preprepared common adsorbents were used, which are activated carbon, alumina, and white eggshells in a batch mode unit. The results obtained from the study showed that 5 was the best acidic function $(\mathrm{pH})$ for removing vanadium and cadmium by using all adsorbent materials, while the acidic function with a value of 6 was the optimum in the case of nickel. Likewise, the equilibrium concentration with the activated carbon only reached $150 \mathrm{ppm}$ for vanadium and cadmium and $100 \mathrm{ppm}$ for elemental nickel. The nickel equilibrium concentration reached 90 and $75 \mathrm{ppm}$ using alumina and white eggshells respectively. The equilibrium concentrations of vanadium were 100 for the rest of the substances. The results obtained also showed that increasing the agitation speed leads to enhancing the removal efficiency within less than $400 \mathrm{rpm}$ and with a best contact time was 150 minute under ambient temperature and with an amount of adsorbent ranged between $0.3-0.7 \mathrm{~g}$ of different adsorbent.
\end{abstract}

Keywords: Activated carbon, Adsorption, Refinery wastewater, Heavy metals, Batch mode adsorption unit.

\section{Introduction}

Pollution is one of the major problems facing humans and environments, especially after the technological development accompanying contemporary life, and pollution occurs in its various forms, whether it is air, water or soil pollution. As a result of the presence of some harmful organic and inorganic materials or because of the increase or decrease in the proportions of some basic components in the environment from the proportions natural phenomena, and this happens consequently of human interventions or some natural phenomena [1]. Water pollution takes many forms, such as toxicity with inorganic wastes, pesticides or detergents, or pollution resulting from food enrichment, thermal pollution, or pollution with oil materials resulting from various oil activities such as extraction residues, oil refineries and other types of pollution [2]. The problem of oil pollution arose because of the rapid technical development in the oil industry, which resulted in an increase in its production and an irregular increase in its use. Therefore, the refineries production of oil derivatives doubled several times during the second half of the last century, and consequently, the introduction of oil pollutants to the environment, Contamination by oil and its derivatives resulting from different oil refineries is characterized by being one of the most

*Corresponding Author: zainabi280@gmail.com 
dangerous types of water pollution due to its rapid spread, as it reaches nearly a distance of $700 \mathrm{~km}$ from the area of its discharge [4]. Lead, cadmium, nickel, vanadium, and iron are among the heavy metals found in most Iraqi crude oil types in particular, and therefore oil refinery wastes contain these heavy metals in different proportions depending on the modernity of the refinery and the method of treatment and disposal of liquid wastewaters [5]. There are several current methods used to treat wastewater contaminated with heavy metals, among all the previous techniques and methods, adsorption is considered one of the most important of these technologies due to its high efficiency in this field and the simplicity of the technology used for this purpose compared to other methods, as well as its appropriate economic cost. Most conventional adsorption systems use activated carbon as the adsorbent [6]. The reason for this is due to the large numbers of surface pores that make the surface area subjected to adsorption wide relative to the actual volume affecting as well as the possibility of reactivating it, as the efficiency of the adsorption decreases over time, and thus requires replacing or regenerating the activated carbon by heat or chemicals. From another side the adsorption capacity decreases with increasing temperature [7]. The determinants of the use of activated carbon prompted many researchers to prepare new adsorbent materials for use in various research purposes. The aim of preparing these materials was to obtain adsorbent materials that could be used in special applications or adsorbent materials from cheap natural sources [8].

Other materials were used due to their properties and advantages such as adsorption media for heavy elements from polluted aqueous solutions and waste water, where flying dust was used [9], as adsorbent for cadmium and silver ions, and alumina silicates [10] to remove lead, cadmium, cobalt and nickel elements, and nanocomposite polymeric materials [11]. On the other hand, agricultural wastes were used on a large scale in the treatment of water contaminated with heavy elements, either directly or indirectly, by using it as a raw material for the preparation of activated carbon. An example of agricultural waste used as an adsorbent for heavy metals is banana peels [17], to remove copper and lead and tea leafs [18] to remove nickel element. The present study aims to investigate the efficiency of the batch adsorption process of three adsorbents, which are activated carbon prepared from cigarette butts, alumina prepared from aluminum foil and white eggshells to remove three heavy metals, namely, vanadium, nickel and cadmium from wastewater discharged from three Iraqi oil refineries, as well as studying the isothermal, kinetic and thermodynamic properties of the adsorption process.

\section{Experimental Work:}

\subsection{Adsorbent Materials:}

Three types of adsorbent materials were used to study the process of treating simulating wastewater from Iraqi oil refineries. These materials were activated carbon and alumina, which were prepared previously from low-cost materials (i.e. Cigarette Butte and Aluminum Foil respectively) and white eggshells collected from continuous domestic use.

\subsection{Samples of the Study and Their Collecting:}

Samples were collected from discarded water of three Iraqi refineries which were Al-Shuaiba refinery in Basra governorate, the Kirkuk refinery in Kirkuk governorate, and Al-Doura refinery in the governorate of Baghdad during 2019-2020 by taking a clean and sterile one-liter beaker and submerging it in the wastewater collection basin from the refinery, taking the sample and covering it tightly, then wrapping it 
with aluminum foil and recording the sample number and the date of its withdrawal.

\subsection{Laboratory Tests of Collecting Samples:}

Three types of laboratory tests were performed on the collected samples, namely, physical, chemical and biological investigations. All checks were conducted in the oil refinery laboratories (except for the March and April samples, which were delayed due to the circumstances that accompanied the closure of government and public institutions due to the COVID-19 pandemic and were examined with the May samples).

\subsection{Laboratory Tests of Heavy Metals:}

The concentrations of heavy elements were determined using the method described by [14], $40 \mathrm{ml}$ of wastewater samples were measured for the refineries and placed in a centrifuge at a speed of $6000 \mathrm{rpm}$ for one hour to get rid of any suspended impurities or organic matter. The supernatant was extracted from the test tube, 8 $\mathrm{ml}$ was withdrawn from it with a glass pipette, and then $4 \mathrm{ml}$ of concentrated nitric acid $\left(\mathrm{HNO}_{3}\right)$ was added and heated using a hot plate. Before drying the sample, the same volume of nitric acid was added and the heating process continued until a precipitate was formed in the beaker. After that, deionized water was added to the precipitate and the volume was completed to $40 \mathrm{ml}$. The solution was filtered using filtration paper and a vacuum filtration device. Finally, the samples were examined with an atomic absorption spectrophotometer (AAS) to determine the amount of heavy metals in them and according to the calibration curves previously prepared for each one of the metals. Concentration of vanadium, cadmium, and nicle in all tested samples were measured.

\subsection{Preparation of Stock Solutions:}

Stock solutions were prepared by dissolving calculated amounts of vanadium, nickel and cadmium salts respectively in one liter of deionized water to obtain a storage solution of $1000 \mathrm{mg} / \mathrm{l}$. The dissolution process was carried out at laboratory temperature and using hotplate magnetic stirrer for 20 minutes to ensure optimum mixing. The stock solution was diluted to the required concentrations in preparing the solutions used in the subsequent experiments.

\subsection{Calibration Curves of Heavy Metals Studied:}

Calibration curves for vanadium, nickel and cadmium metals were prepared using specific concentrations previously of these salts and was examined by AAS device that relies on the absorbance principle at a specific length for each element. The calibration curve is used to find the concentrations of the metal ions after the adsorption process, where the residual concentration after treatment is estimated of the absorbance from the device. Calibration curves performed at wavelengths of 306.6, 351.5, and $326.1 \mathrm{~nm}$ for vanadium, nickel, and cadmium ions, respectively.

\subsection{Experiments of Adsorption:}

Adsorption experiments were performed using simulated solutions prepared from dilution of the stock solution to a specific concentration using deionized water in a water bath shaker. $100 \mathrm{ml}$ of aqueous solution containing heavy metals (vanadium, nickel, cadmium) was placed in $150 \mathrm{ml}$ glass flasks and covered with aluminum foil tightly to avoid spillage during the shaking process. The acidic function of the solution was controlled using solutions of $0.1 \mathrm{M}$ hydrochloric acid and $0.1 \mathrm{M} \mathrm{NaOH}$. Also the specified amounts of adsorbent (activated carbon, alumina, eggshells) were put with the aqueous solution of heavy metals. After preparing the samples in the flaxes and 
completing their installation in the shaker, and by setting the temperature of the shaker, the treatment process begins with adjusting the agitation speed and continues until the end of the specified period of time. The operating conditions used ranged between (1-8), (1-400) $\mathrm{mg} / \mathrm{l}, \quad(100-400)$ rpm, (0.01-1) g, (10-180) minutes, (20-50) ${ }^{\circ} \mathrm{C}$ for the acidic function, initial concentration, agitation speed, amount of adsorbent, contact time, and temperature respectively. After the expiration of the time period, the shaker is stopped, the flasks are extracted, and the process of filtering samples by Whiteman Filter Paper 41 and using a vacuum filtering kit, after the adsorbent material is isolated from the solution. The sample is examined by the AAS device to find out the concentration of the remaining heavy metals and to determine the percentage of removal $(\% R)$, and the capacity of the adsorbent of the adsorption (q) using Equations (1) and (2), respectively.

$\% R=\frac{C_{i}-C_{f}}{C_{i}} \times 100$

$q=\frac{V}{m} \times\left(C_{i}-C_{f}\right)$

Where: $R$ : is the percent removal of heavy metal ions,$q$ : is the capacity of adsorption by adsorbent media $(\mathrm{mg} / \mathrm{g}), C_{i n}$ and $C_{f}$ : initial and final concentrations of heavy metal ions respectively $(\mathrm{mg} / \mathrm{l}), \mathrm{m}$ : mass of adsorbent used for adsorption $(g)$ and $V$ : volume of solution (l).

\section{Results and Discussions}

\subsection{Effect of Acidic Function (pH):}

The effect of the acidic function $(\mathrm{pH})$ on removing heavy metal ions (vanadium, nickel, and cadmium) from simulated aqueous solution using activated carbon, alumina and white eggshells was studied with a range between (18 ) and the rest of the other operational factors were kept at optimum values. Fig.1 to Fig.3 show that the relationship between the acidic function of the solution containing heavy metals and the removal percentage is direct relation up to the value of $\mathrm{pH}=5$ or $\mathrm{pH}=6$, after which the removal efficiency increases sharply in the case of eggshells and for all metals until reach the full percentage.

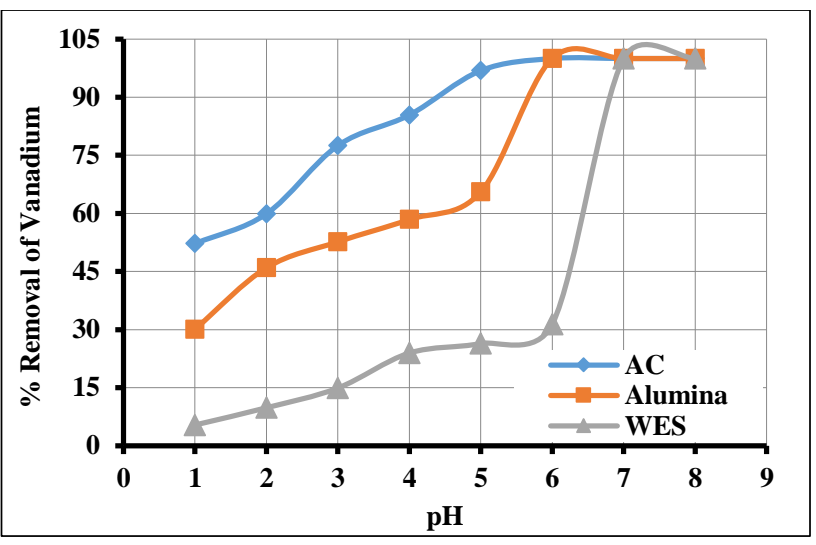

Figure 1. Effect of Acidic Function $(\mathrm{pH})$ on \% Removal of Vanadium Using Three Types of Adsorbents

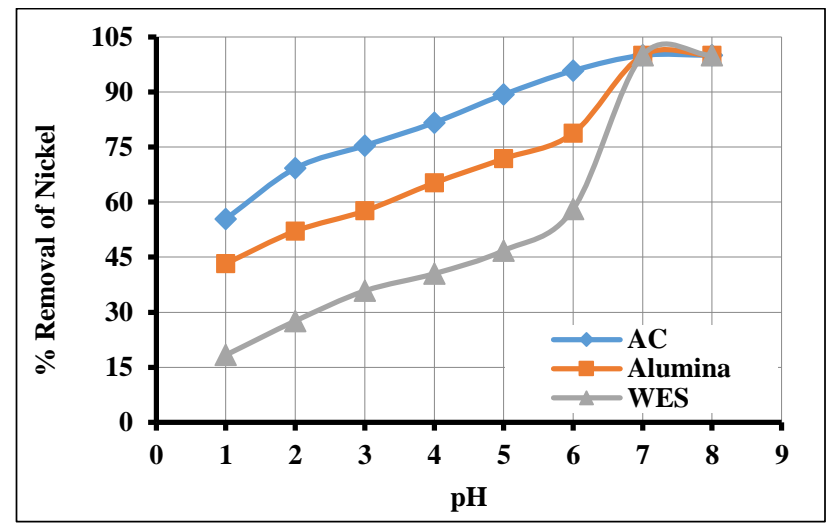

Figure 2. Effect of Acidic Function (pH) on \% Removal of Nickel Using Three Types of Adsorbents

The reason for this result may be that the adsorption of heavy metal ions decreases at lower $\mathrm{pH}$ values due to the competition of positive hydrogen ions $\left(\mathrm{H}^{+}\right)$for metal ions for the adsorption sites, which increases with the decrease in the value of the acidic function. But with the increase in the value of the acidic function, the positive hydrogen ions $\left(\mathrm{H}^{+}\right)$will decrease due to the increase in the concentration of the negative hydroxide ions $\left(\mathrm{OH}^{-}\right)$, which will reduce the competition and thus the adsorption 
of vanadium, nickel and cadmium ions will increase and their concentration in the solution will increase and the removal percentage will increase.

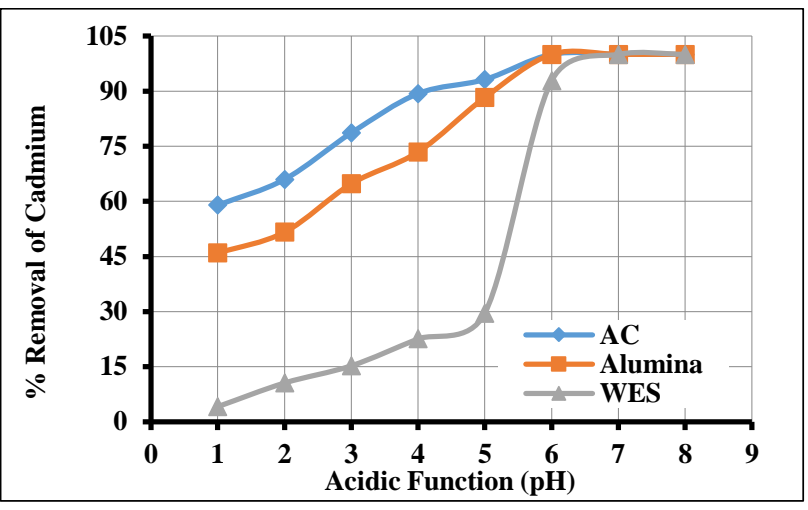

Figure 3. Effect of Acidic Function (pH) on \% Removal of Cadmium Using Three Types of Adsorbents

The acidic function $(\mathrm{pH})$ factor is considered as one of the most important factors that must be studied and verified in any adsorption process to find out the ideal value that separates adsorption from precipitation. Therefore, the sharp and rapid increase in the removal efficiency at $\mathrm{pH}$ values greater than 5 or 6 can be attributed to the precipitation process that occurs, which reduces the concentration of heavy metal ions in the solution, but they precipitate at the bottom of the beaker and thus increase the removal percentage. Thus, the optimum acidic function for the adsorption of vanadium and cadmium is 5 and nickel is 6 .

\subsection{Effect of Initial Concentration $\left(C_{0}\right)$}

The change in the efficiency of the process of treating solutions contaminated with heavy metals has been studied as a result of the effect of the initial concentration within the range between (1-400) ppm for vanadium and cadmium and (1-250) ppm for nickel and under optimal conditions for the rest of the other design conditions. It is noticed from the obtained results that the relationship between the two variables is in an inverse relationship, as the percentage removal decreases gradually with the increase of the initial concentration value for all heavy metal ions in general and for all adsorbent materials. The activated carbon, alumina, and white eggshells, which represent the adsorption media in this study, have a constant surface area. This means that the number of adsorbable ions on the surface of the adsorbent is limited. Therefore, the concentration of the contaminant, as it increases, means an increase in the ions of those metals because the volume of the solution is constant, that is, the increase in competition between ions of heavy metals for the same number of active sites on the surface of the adsorbent. This, in turn, will reduce the number of ions adsorbed and increase the number of heavy metal ions in the contaminated solution and thus the treatment efficiency will decrease. This is evident in Fig .4, 5,6 which represent the relationship of the initial concentration to the percentage removal of vanadium, nickel, and cadmium, respectively.

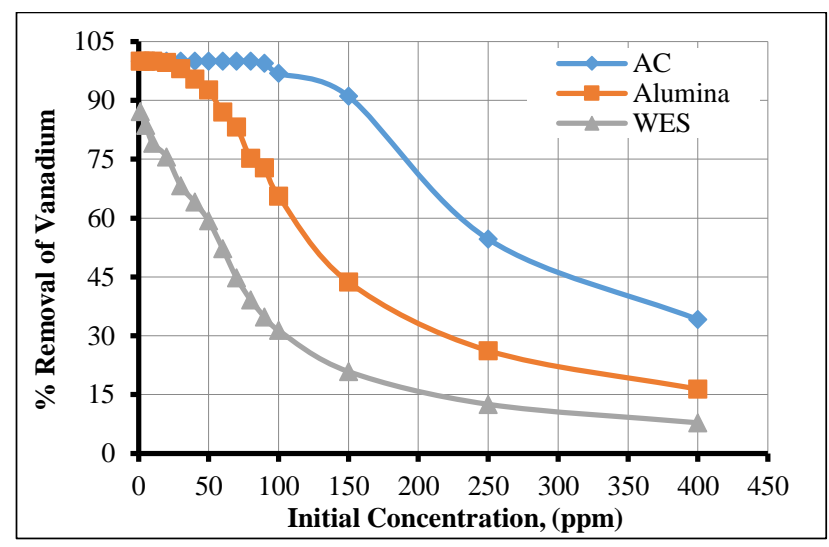

Figure 4. Effect of Initial Concentration on \% Removal of Vanadium Using Three Types of Adsorbents

\subsection{Effect of Agitation Speed $\left(A_{s}\right)$ :}

By keeping the rest of the other operational variables at optimal values, the nature of the relationship between the percentage of removal of vanadium, nickel and cadmium elements on the one hand and the speed of agitation using adsorbents (activated carbon, alumina and white 
eggshells) within the range of (100 to $500 \mathrm{rpm}$ ) was investigated.

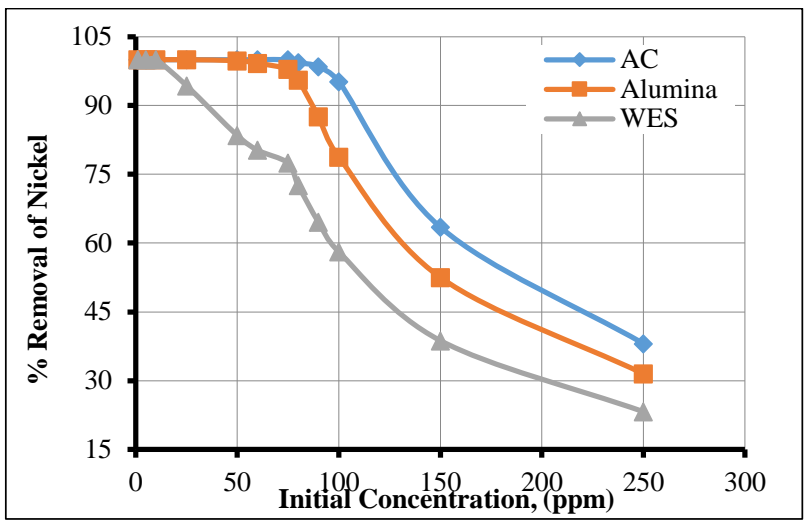

Figure 5. Effect of Initial Concentration on \% Removal of Nickel Using Three Types of Adsorbents

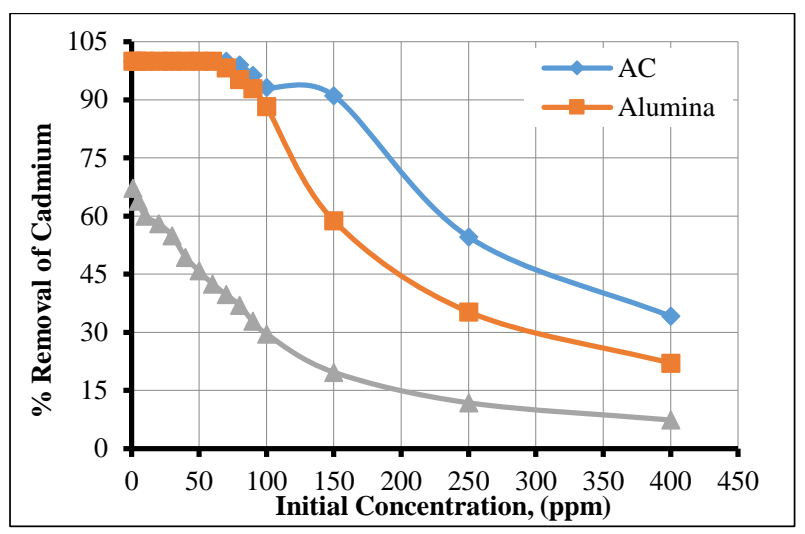

Figure 6. Effect of Initial Concentration on \% Removal of Cadmium Using Three Types of Adsorbents

It is noticed from Fig.4-7, 4-8 and 4-9 that the relationship between the agitation speed and the percentage removal percentage is direct relation using all the adsorbent materials. The reason for this result is due to diffusion as the increased diffusivity of heavy metal ions in the solution can be attributed to the increased agitation speed, which in turn will lead to an increased chance of vanadium, nickel and cadmium ions reaching the active sites on the surface of activated carbon, alumina and white eggshells which will increase the percentage of adsorption. Or, an increase in the agitation speed may lead to the destruction of the boundary layers formed around the adsorbent molecules, and thus the number of heavy metal ions arriving at the active sites on the surface subject to adsorption will increase, thus their number in the solution will increase and the treatment efficiency will increase. Fig.7,8 and 9 show the relationship between the percentage of removal of vanadium, nickel and cadmium using all adsorbents. It is also noticed from the above figures that the removal efficiency after reaching $400 \mathrm{rpm}$ remains constant and does not change.

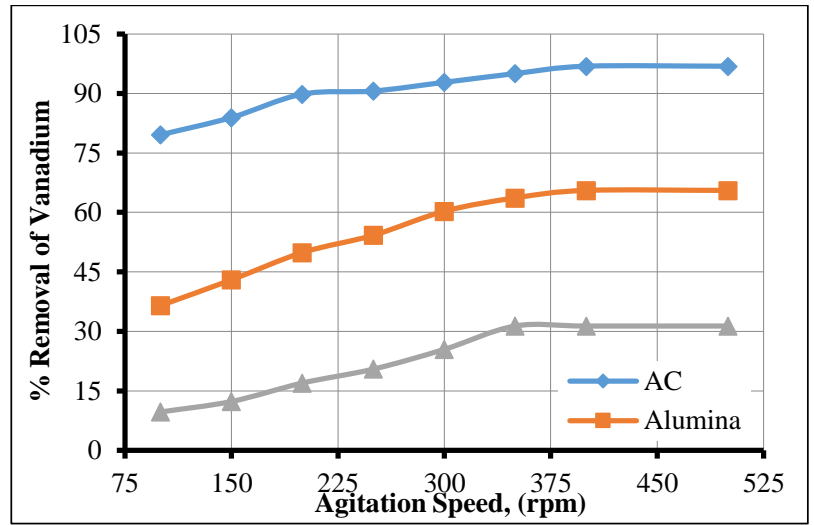

Figure 7. Effect of Agitation Speed on \% Removal of Vanadium Using Three Types of Adsorbents

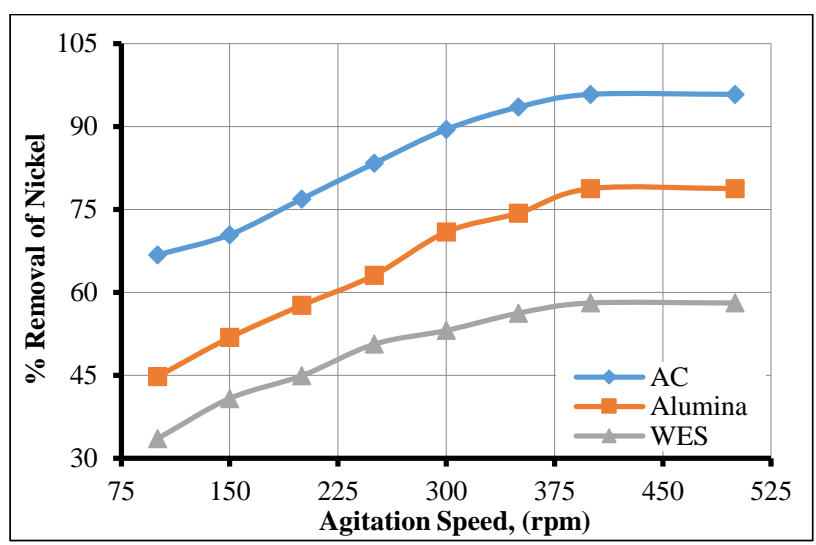

Figure 8. Effect of Agitation Speed on \% Removal of Nickel Using Three Types of Adsorbents 


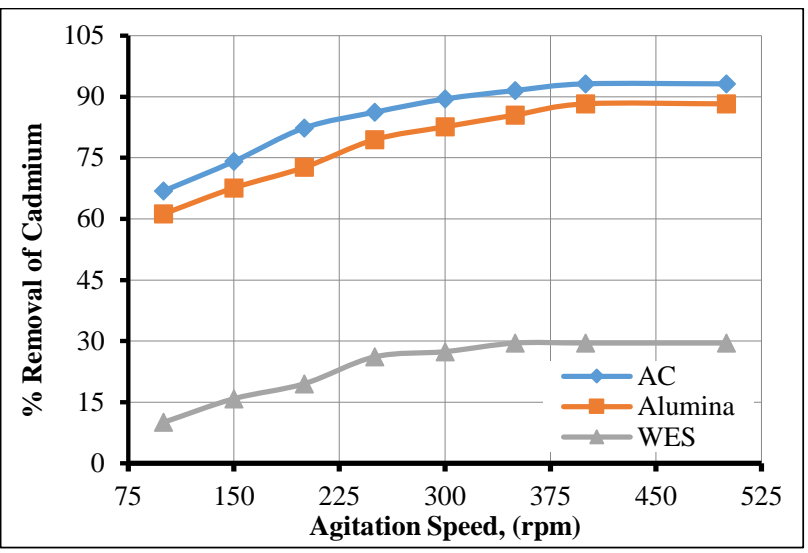

Figure 9. Effect of Agitation Speed on \% Removal of Cadmium Using Three Types of Adsorbents

The reason for the confirmation of the removal percentage may be due to the generation of repulsive forces between the molecules of the adsorption media as a result of the high speed that generates severe turbulence with which the heavy metal ions are not able to reach the effective sites on the surface of the adsorption media. This will make the number of ions in the solution increase as a result of the constant surface area of the adsorbent material, which will reduce the efficiency of the treatment process. It can be concluded that $400 \mathrm{rpm}$ is the optimum agitation speed for obtaining maximum removal efficiency.

\subsection{Effect of Adsorbent Media Amount (m)}

The effect of the adsorbent amount (activated carbon, alumina, white eggshell) on the efficiency of adsorption of heavy metal ions in aqueous solutions simulating the wastewater of Iraqi petroleum refineries was studied through the use of different quantities of the adsorbent material ranged between (0.01-1) $\mathrm{g}$ and the rest of the variables were fixed at optimum values. It was found through this study that using an amount of $0.3 \mathrm{~g}$ of activated carbon to treat solutions contaminated with vanadium, nickel and cadmium, the removal percentage reaches the maximum value of $100 \%$, while the contaminated aqueous solutions need $0.4 \mathrm{gm}$ of vanadium and nickel ions and $0.5 \mathrm{~g}$ of cadmium ions to obtain complete removal, whereas $88.3256 \%, 77.4542 \%$ and $69.3825 \%$ are the maximum removal achieved by using $0.6 \mathrm{~g}$, $0.6 \mathrm{~g}$, and $0.8 \mathrm{~g}$ egg white shells to treat water contaminated with vanadium, nickel and cadmium respectively. This can be attributed to the active sites on the surface of the material that are available for the adsorption of heavy metal ions, which are higher in activated carbon and alumina compared to white eggshells.

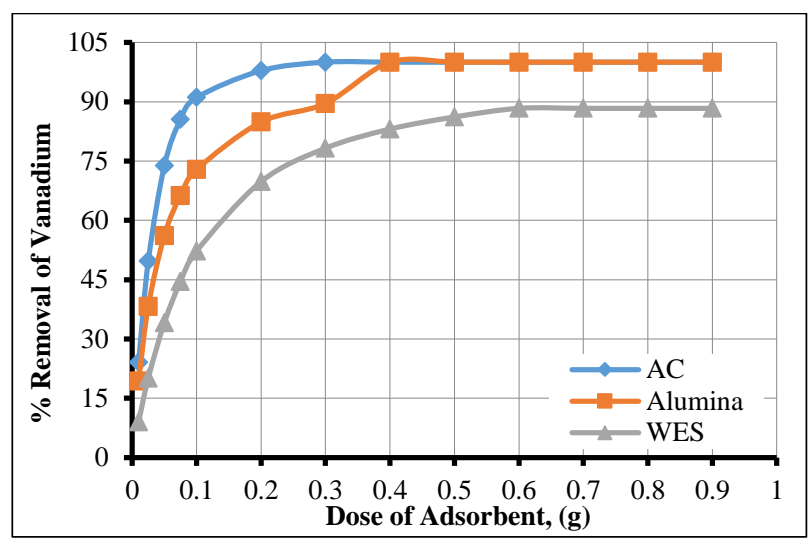

Figure 10. Effect of Adsorbent Dose on \% Removal of Vanadium Using Three Types of Adsorbents

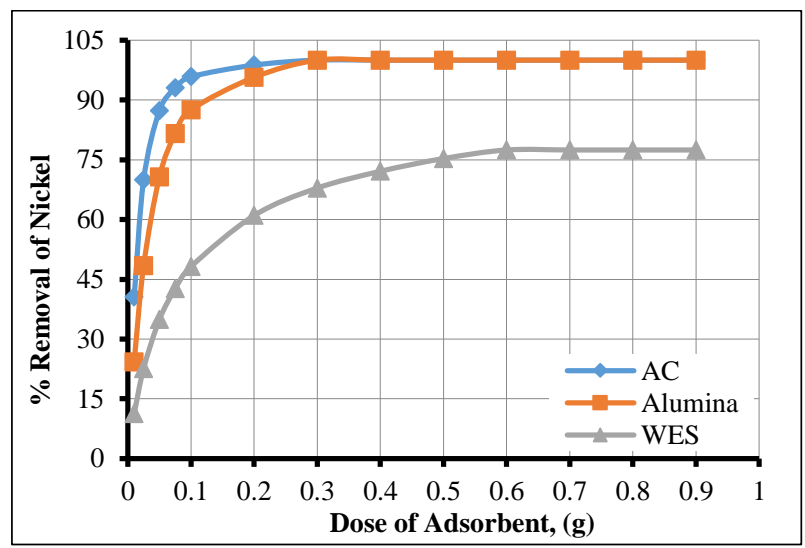

Figure 11. Effect of Adsorbent Dose on \% Removal of Nickel Using Three Types of Adsorbents

When using larger amounts of material, the mass of adsorbent central desorption increases and thus increasing the surface area thereby increasing the effective sites. This, in turn, will increase the chance of adsorption of heavy metal ions and thus increase the adsorption 
efficiency.Fig.10, 11 and 12 show the relationship of adsorption quantity and adsorption efficiency for vanadium, nickel and cadmium ions respectively.

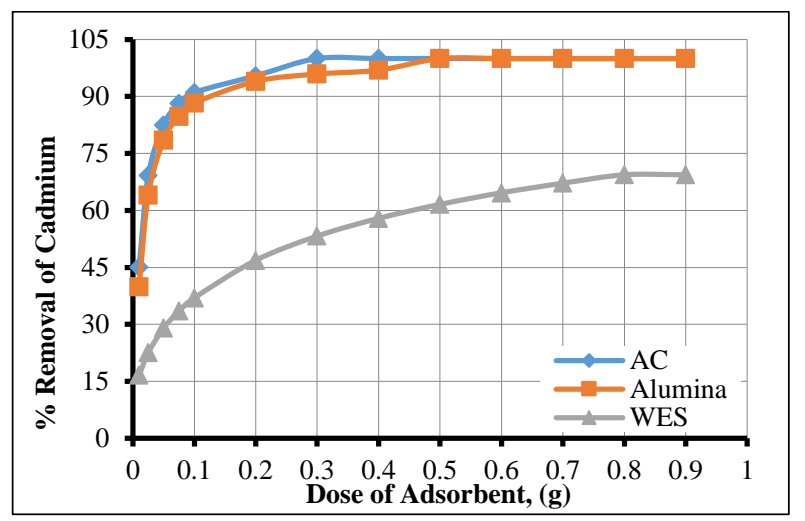

Figure 12. Effect of Adsorbent Dose on \% Removal of Cadmium Using Three Types of Adsorbents

\subsection{Effect of Contact Time ( $t$ )}

An important factor that must be taken into consideration when designing adsorption experiments is the contact time. To study the extent to which the treatment efficiency was affected by the contact time, vanadium, nickel and cadmium adsorption experiments were conducted using activated carbon, alumina and white eggshells with time periods ranging between (10-180) minutes, with the rest of the variables being fixed at the optimum values. Where the time required for the system to reach equilibrium is known, and any time period after that is considered ineffective. The results obtained from practical experiments showed that the percentage of heavy metals removal using adsorbent materials is directly proportional to the contact time and that the highest processing efficiency is obtained at 150 minutes of activated carbon, while the treatment efficiency reaches its maximum value before that by using alumina and white eggshells. But after increasing the contact time beyond two and a half hours, the removal efficiency does not change and remains constant for all metals and adsorbed materials, and this is shown in Fig.
13, 14 and 15. The increase in contact time with constant other operational factors means increasing the period of time of the contaminant ions staying in contact with the adsorption medium, which means increasing the chance of those ions reaching the effective sites on the surface of the adsorbent, and this, in turn, will lead to the adsorption of a greater number of ions, and thus their number in the solution decreases to, increases the removal efficiency. When the adsorption surface reaches a saturation state, the increase in time will not affect the adsorption efficiency as a result of the adsorption ions being filled with those active sites and the absence of any other vacancies.

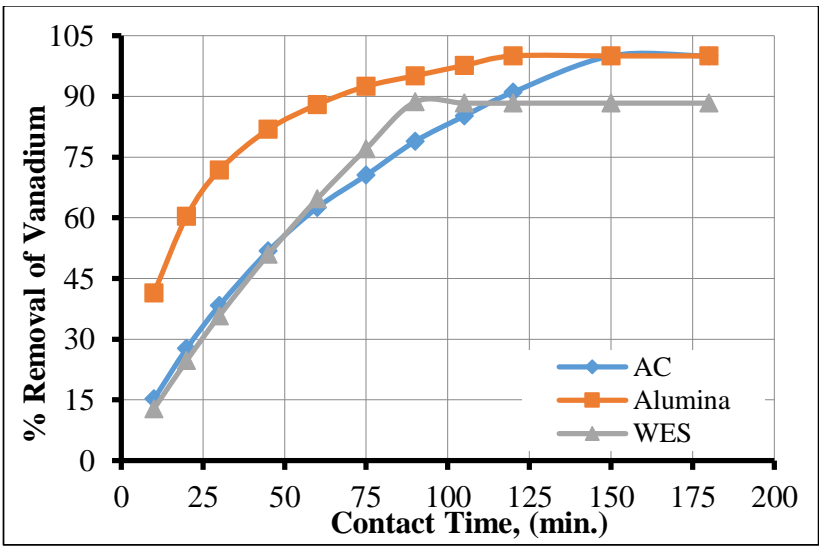

Figure 13. Effect of Contact Time on \% Removal of Vanadium Using Three Types of Adsorbents

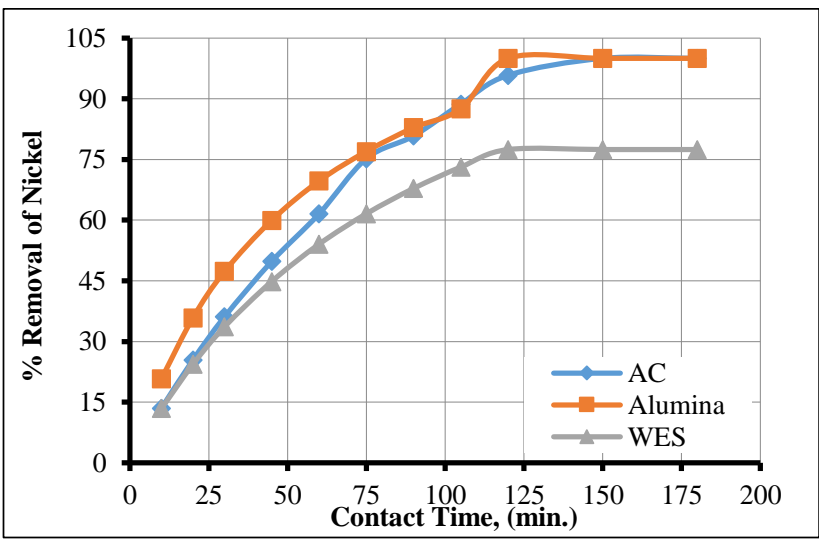

Figure 14. Effect of Contact Time on \% Removal of Nickel Using Three Types of Adsorbents 


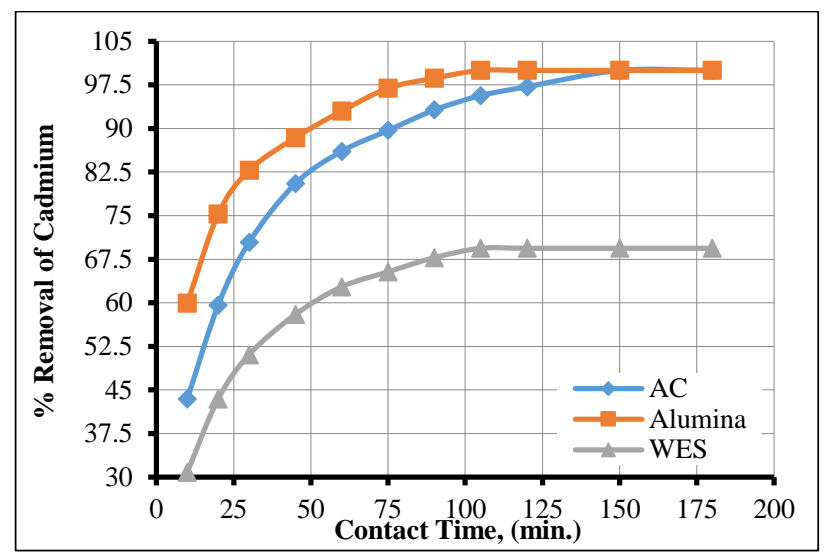

Figure 15. Effect of Contact Time on \% Removal of Cadmium Using Three Types of Adsorbents

Therefore, it can be considered that 150, 120, 90 minutes is the time required to reach the equilibrium state of activated carbon, alumina and egg white shells, respectively.

\subsection{Effect of Temperature (T)}

Fig.16, 17 and 18 show the effect of temperature on the efficiency of the removal of vanadium, nickel and cadmium using activated carbon, alumina, and white eggshells in the batch mode adsorption unit. It is noticed from the figures above that the maximum removal is achieved at the lower temperature, that is, the inverse relationship between temperature and removal efficiency for all heavy metals investigated and for all adsorbents used. Thus the adsorption process is exothermic and that an increase in temperature leads to a breakdown of the bonds that connect the functional groups present in the active sites spread over the surface of activated carbon, alumina, and white eggshells with vanadium, nickel, and cadmium ions, as a result, it is released and returned to the solution again, reducing the removal efficiency. The maximum removal obtained at $20{ }^{\circ} \mathrm{C}$ using activated carbon and alumina was $100 \%$ for all the studied elements, while the eggshell efficiency at $20^{\circ} \mathrm{C}$ was $88.6256 \%$ for vanadium, $77.4542 \%$ for nickel, and $69.3825 \%$ for cadmium. The reason for this is the higher surface area with respect to activated carbon and alumina compared to the eggshells.

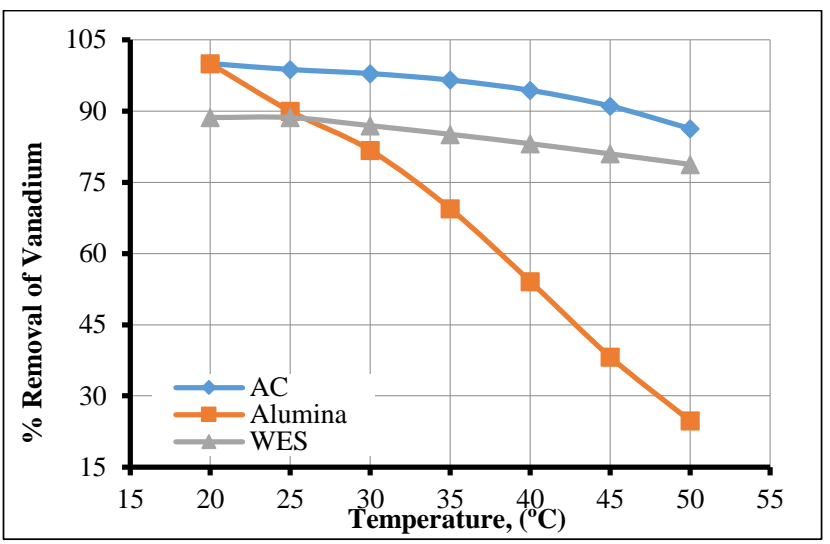

Figure 16. Effect of Temperature on \% Removal of Vanadium Using Three Types of Adsorbents

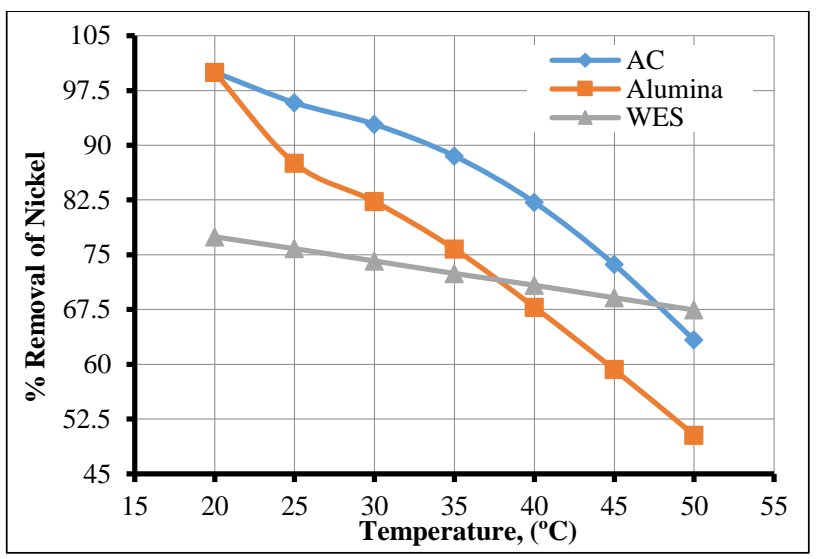

Figure 17. Effect of Temperature on \% Removal of Nickel Using Three Types of Adsorbents

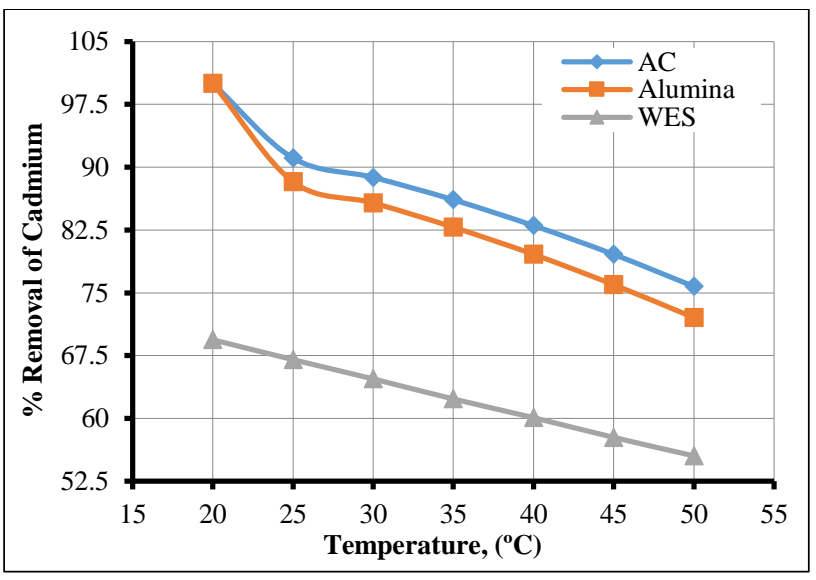

Figure 18. Effect of Temperature on \% Removal of Cadmium Using Three Types of Adsorbents 


\section{Conclusions:}

- The adsorption technique has shown high efficiency for treating liquid wastewaters of Iraqi petroleum refineries, especially the heavy metals, the main pollutants in petroleum wastewaters, which are vanadium, nickel and cadmium.

- Activated carbon and alumina were the best adsorption materials used, as the treatment efficiency reached the ideal efficiency for all metals, while the treatment efficiency using eggshells was $88.3256 \%, \quad 77.4542 \%$, $69.3825 \%$ for vanadium, nickel and cadmium, respectively.

- The results showed that the optimum operating conditions that achieve the highest removal efficiency were as follows: the acidic function 5 for vanadium and cadmium and 6 for nickel and for all adsorption materials used, agitation speed $400 \mathrm{rpm}$ for all metals and almost all adsorption materials.

- The equilibrium time was 150 minutes using activated carbon, 120 minutes using alumina for all metals, while it was 90, 120 and 105 minutes using eggshells for vanadium, nickel and cadmium respectively.

- The equilibrium concentration of vanadium was 100 ppm using activated carbon and alumina and 60 ppm using eggshells, while the equilibrium concentration of nickel was 100, 90, and 75 ppm using activated carbon, alumina and eggshells respectively, but for cadmium the equilibrium concentration using activated carbon, alumina and eggshells were 150,100 , and 80 respectively.

- For vanadium and nickel, the best amount of adsorbent materials was $0.3,0.4,0.6 \mathrm{~g}$ for activated carbon, alumina and eggshells, while for cadmium the quantity for activated carbon, alumina and eggshells was 0.3, 0.5, and $0.8 \mathrm{~g}$, respectively.

- The removal efficiency of all metals and with the use of all adsorption materials was inversely proportional with temperature as the highest removal efficiency was at $20{ }^{\circ} \mathrm{C}$ and the lowest removal efficiency at $50{ }^{\circ} \mathrm{C}$.

\section{Conflict of Interest}

The authors would like to declare that there are no conflicts regarding the publication of this article.

\section{References:}

1. Vilardi G., Di Palma L. and Verdone N., (2018), "Heavy metals adsorption by banana peels micro-powder: Equilibrium modeling by non-linear models", Chinese Journal of Chemical Engineering, Volume 26, Issue 3, pp: 455-464. March. https://doi.org/10.1016/j.cjche.2017.06.02

\section{$\underline{6}$}

2. Zhongjun X., Na L., Hongxiang H., Jieying H., Kianpoor K. Y., Xiuyuan W., Nian X., Xiaofei F. and Linchuan Z., (2019), "Adsorption of Cd(II) in water by mesoporous ceramic functional nanomaterials", Royal Society open science, Volume 6, Issue 4, 11 Pages. http://doi.org/10.1098/rsos.182195

3. Vo T. S., Hossain M. M., Jeong H. M. and Kim K., (2020), "Heavy metal removal applications using adsorptive membranes", Nano Convergence, Volume 7, Article number

36.

https://doi.org/10.1186/s40580-02000245-4

4. Wake H., (2005), "Oil refineries: a review of their ecological impacts on the aquatic environment", Estuarine, Coastal and Shelf Science, Volume 62, Issues 1-2, January, Pages 131-140. https://doi.org/10.1016/j.ecss.2004.08.013

5. Allafta H. and Opp C. (2020), "Spatiotemporal variability and pollution sources identification of the surface sediments of Shatt Al-Arab River, Southern Iraq", Scientific reports, Volume 10, Issue 1, 6979. https://doi.org/10.1038/s41598-02063893-w

6. De Gisi S., Lofrano G., Grassi M. Notarnicol M., (2016), "Characteristics and adsorption capacities of low-cost 
sorbents for wastewater treatment: A review", Sustainable Materials and Technologies, Volume 9, September, Pages $10-40$. https://doi.org/10.1016/j.susmat.2016.06.0 $\underline{02}$

7. Alalwan H. A., Kadhom M. A. and Alminshid Al. H., (2020), "Removal of heavy metals from wastewater using agricultural byproducts", Journal of Water Supply: Research and Technology-Aqua, Volume 69, Issue 2, pp: 99-112. https://doi.org/10.2166/aqua.2020.133

8. Nasira W., Muhammad S., Muhammad I., Akhtar M., Muhammad S., Manzar Z. and Amir W., (2019), "Synthesis, Characterization, and Applications of Silk/Bentonite Clay Composite for Heavy Metal Removal from Aqueous Solution", Frontiers in Chemistry, Volume 7, Article number 654, 12 Pages. https://doi.org/10.3389/fchem.2019.00654

9. Nguyen T. C., Tran T. D. M., Dao V. B., Vu Q.-T., Nguyen T. D., Thai H., (2020), "Using Modified Fly Ash for Removal of Heavy Metal Ions from Aqueous Solution", Journal of Chemistry, Volume 2020, Article ID 8428473, 11 Pages. https://doi.org/10.1155/2020/8428473

10. Treto-Suárez M. A., Prieto-García J. O., Mollineda-Trujillo Á., Lamazares E., Hidalgo-Rosa Y. and Mena-Ulecia K., (2020), "Kinetic study of removal heavy metal from aqueous solution using the synthetic aluminum silicate". Scientific Reports, Volume 10, Article number: 10836. https://doi.org/10.1038/s41598$\underline{020-67720-0}$
11. Zhao G., Huang X., Tang Z., Huang Q., Niu F. and Wang X., (2018), "Polymerbased nanocomposites for heavy metal ions removal from aqueous solution: a review", Polymer Chemistry, The Royal Society of Chemistry, Issue 26, 9, pp: 3562-3582.

https://doi.org/10.1039/C8PY00484F

12. Vilardi G., Di Palma L. and Verdone N., (2018), "Heavy metals adsorption by banana peels micro-powder: Equilibrium modeling by non-linear models", Chinese Journal of Chemical Engineering, Volume 26, Issue 3, pp: 455-464. March. https://doi.org/10.1016/j.cjche.2017.06.02 6

13. Malkoc E. and Nuhoglu Y., (2005), "Investigations of nickel (II) removal from aqueous solutions using tea factory waste", Journal of Hazardous Materials, Volume 127, Issues 1-3, pp: 120-128. 9 December.

https://doi.org/10.1016/j.jhazmat.2005.06. $\underline{030}$

14. Ali G. A. A., Ibrahim S. A. and Abbas M. N., (2021), "Catalytic Adsorptive of Nickel Metal from Iraqi Crude Oil using non-Conventional Catalysts", Innovative Infrastructure Solutions, Volume 6, Article number: 7, 9 pages. https://doi.org/10.1007/s41062-02000368-x. 